\title{
Squeezing the Toothpaste Tube: Will Tackling Court Delay Result in Pre-Court Delay in its Place?
}

\section{Bridget McKeigue}

Bridget McKeigue is a senior lecturer in social work at Anglia Ruskin University in Cambridge, and has been engaged in research into decision-making and delay in care proceedings for several years. Prior to taking up this post she was a Child Protection Co-ordinator in a Social Services Department and as a Child Care Trainer. Prior to this she worked for many years as a children and family social worker.

\section{Chris Beckett}

Chris Beckett is also a senior lecturer in social work at Anglia Ruskin University in Cambridge. $\mathrm{He}$ has been involved in research into decision-making and delay in care proceedings since 1999. Before taking up his current post he was the team manager of a children and families social work team. His publications include a textbook on child protection. He is a member of the editorial board of the journal Ethics and Social Welfare.

Correspondence to Chris Beckett, Webb Building, Anglia Ruskin University, East Road, Cambridge CB1 1PT, UK. E-mail: Chris.Beckett@anglia.ac.uk

This is a pre-copy-editing, author-produced PDF of an article accepted for publication in British Journal of Social Work following peer review. The definitive publisher-authenticated version, McKeigue, B., \& Beckett, C., 2008. Squeezing the toothpaste tube: will tackling court delay result in pre-court delay in its place?. British Journal of Social Work, (2010) 40(1), pp.154-169 is available online at: http://bjsw.oxfordjournals.org/content/40/1.toc

\section{Summary}

This article is the first to report on a study that tracked all the care proceedings initiated by an English local authority over a one-year period, obtaining factual information and opinions from the social workers involved in the case through the use of guided interviews. The main objective of the researchers was to contribute to understanding of ways in which children are kept waiting in difficult and insecure situations for decisions to be made about their long-term future. This article focuses in particular on the problem of delays that occur prior to a case coming into the court arena. The article offers evidence that current initiatives to reduce court delay may have the unintended result of increasing pre-court delay.

Keywords: Care proceedings, court delay, pre-court delay, social work decision making

\section{Introduction}

This article describes a study that looked at all the care proceedings undertaken by one local authority over the period of a year. (For international readers: care proceedings are court proceedings in which local authorities apply for parental responsibility over children.) The study was prompted by our concerns (e.g. Beckett, 2001; McKeigue and Beckett, 2004) and those of many others over a long period (e.g. Goldstein et al., 1980; Steinhauer, 1991; Thomas et al., 1993; Booth, 1995; Hunt et al., 1999) that it is not good for children to have to endure protracted periods 'in limbo' while their future is decided. We explored this in a previous study in another local authority area (Beckett and McKeigue, 2003) in which we looked at children's cases that had taken an exceptionally long time. The present study was more comprehensive (all proceedings, not just long ones) and it involved collecting data during the process (rather than retrospectively) about what happened to the children and the way in which decisions about their future were made. We intend in future articles to discuss what happens during and after the court process, and to look at possible causes of delay during those periods. Here, however, after outlining the methodology of the study as a whole and reporting on some of our quantitative findings, we intend to focus our discussion on delay and insecurity prior to the proceedings taking place. We will call this 'pre-court delay'. 
This seems to us to be topical at a time when redoubled efforts are being made in the UK to tackle the problem of delay during proceedings (Ministry of Justice, 2008), because we see a danger that bearing down solely on delay within the court process may create additional delay elsewhere in the system, rather in the way that if one squeezes a bulge in a toothpaste tube, it simply creates a new bulge further along. This would be an unintended consequence of a kind familiar to critics of 'targets' in other areas, such as hospital waiting lists (see, for example, recent reports of patients being 'stacked' in ambulances outside accident and emergency units, in order to meet government targets for waiting times within those units: Campbell, 2008). Our findings seem to us to provide evidence that this danger is real. Yet, as other authors have noted (Bishop et al., 1992; McSherry et al., 2004), it does not really matter to children whether the delay is before, after or during the court process: what matters is that children should not spend long periods in a state of insecurity.

\section{Methodology}

The study looked at all the care proceedings initiated over the period 1 April 2004 to 31 March 2005 by a local authority that covers a mixed rural and urban area in England. (Cases were then followed through to their conclusion, meaning that the study itself was ongoing into 2006.) The authority has a centralized system for making decisions about whether a case should be taken into the court arena and was able to notify us of all cases in which this decision was made during the timeframe. Information was gathered by interviewing social workers involved from the outset of proceedings until the final hearing, at approximately threemonth intervals. No social worker refused to take part in the study but the actual number of interviews carried out depended on the length of proceedings and on the time the social worker was able or willing to invest in talking to us. The average duration of proceedings was thirty-five weeks.

The average number of interviews per case was just under three, and interviews usually lasted approximately an hour and a half. Interviews were guided by a detailed proforma that asked for factual information, as well as opinions, on a variety of issues, including: the characteristics of the children and their families and the reasons for their being subject to proceedings; the management of their case by the local authority; the assessment, decisionmaking and legal process; the demands made on the social workers; the eventual outcome; and the impact of the process on the children themselves.

At the analysis stage, we first extracted purely factual and quantitative data (some of which are reported below). We then carried out a qualitative analysis that consisted of (i) preparing a summary of the interviews carried out on each case, and listing the key issues that emerged, (ii) compiling a list of all the key issues identified, grouping these into themes and identifying those themes that had been apparent across a number of cases, and (iii) returning to our case summaries to compile a list of all instances in which our key themes had been apparent. The methodology can be described as a form of 'qualitative thematic analysis' (Seale, 2004, p. 314), and therefore has both the advantages and disadvantages of any qualitative approach. This article will deal primarily with one particular theme, which we identified as 'pre-court delay'. We have selected this theme for our first article because it was prominent in our sample and because, for reasons that we will discuss, it seemed to us to be topical.

\section{Limitations of methodology}

We acknowledge a number of limitations to our approach. First, since we had no access to files, we were completely reliant on the social workers for information. Second, the amount of detail we were able to obtain was limited by the amount of time that social workers were able and willing to make available to us. Third, social workers may have felt inhibited in what they told us. Fourth, the account we will be discussing is based only on the perspective of local authority social workers and we heard nothing, except indirectly, of the views of other participants who would have had their own equally valid perspectives. But we repeat the view that we expressed in a previous article (Beckett et al., 2007, p. 57) that, while the observations of the social workers represent only a partial view (as is the case, of course, with our own analysis and comments), their observations about the shortcomings of the system and the constraints that they work under are likely to be well founded. What they may be less 
strong on is the constraints that others work under, or on what others might see as their own shortcomings. Parents, children's guardians, judges, lawyers and children would all almost certainly have been able to identify instances of poor social work practice that our methodology does not allow us to pick up, but all of these participants would have been much less well placed to recognize and describe to us the constraints and difficulties of the social workers' position. 
Ethical approval for this project was obtained in January 2004 from the Joint Schools Research Ethics Group of Anglia Ruskin University (then Anglia Polytechnic University).

\section{Findings}

There were thirty-eight sets of care proceedings initiated during the year in question. These cases involved a total of fifty-nine children.

\section{Case characteristics and circumstances The children}

Of the fifty-nine children, twenty-two were boys and thirty-seven were girls. Their mean age was four years two months. The age range was between a few days old and fourteen years eight months. Twenty-nine per cent of the children were under one year old.

Fifty-four of the children were white British. Three children were mixed race. Two children were black. No child in this group was identified as having a severe disability, although three children were identified as possibly having some degree of learning disability. In three cases, involving four children, the children had been subject to care proceedings previously.

\section{Parents}

The mean age of mothers was twenty-seven. (Data were missing in two cases.) The mode (the most frequent age, reckoned in whole years) was nineteen (five mothers were nineteen). Data about fathers were not consistently available to us.

The children were in a variety of different family contexts, including families in which the identity and/or whereabouts of the father was not known, and families in which the children had several different fathers. In 71 per cent of cases, the mother was not living with the father of any of the children (cf. Brophy et al., 2003, who found that 85 per cent of parents were not living together at the outset of proceedings.) No child was with a lone-parent father. One child was living with, and had been allegedly mistreated by, a kinship carer with whom she had been placed as a result of previous care proceedings.

\begin{tabular}{|c|c|c|}
\hline Problem & $\begin{array}{c}\text { Number of } \\
\text { family cases } \\
(\mathrm{N}=38)\end{array}$ & $\begin{array}{c}\text { Percentage of family } \\
\text { cases (rounded to nearest } \\
\text { whole number) }\end{array}$ \\
\hline Domestic violence & 21 & 55 \\
\hline Mental health problems (mother) & 18 & 47 \\
\hline Learning disability (mother) & 14 & 37 \\
\hline Drug abuse (mother) & 11 & 29 \\
\hline Drug abuse (mother's partner) & 8 & 21 \\
\hline Alcohol abuse (mother) & 7 & 18 \\
\hline Alcohol abuse (mother's partner) & 6 & 16 \\
\hline Mental health problems (mother's partner) & 6 & 16 \\
\hline Learning disability (mother's partner) & 5 & 13 \\
\hline $\begin{array}{l}\text { Cases where none of the above problems } \\
\text { was identified }\end{array}$ & 3 & 8 \\
\hline
\end{tabular}

Table 1 summarizes the problems in the child's family of origin as identified by social workers (more than one problem might be identified per case; percentages are based on family cases rather than on children). Domestic violence was noted in twenty-one families (55 per cent), making it the single most commonly identified problem out of the categories offered and the only one present in more than half of cases. This finding is similar to that of Hunt et al. (1999), 
who found domestic violence allegations featured in 51 per cent of care proceedings cases. The next most common category was mental health problems (mother), noted in eighteen families, or 47 per cent of cases, again similar to Hunt et al. (1999), who found mental health issues to be present in 48 per cent of cases. The mother's partner was identified as also having a mental health problem in six cases. Table 1 lists categories in descending order of frequency.

Social workers were asked to identify the kinds of concern that led to the initiation of care proceedings. They were told that they could identify more than one and concerns might be based on actual or suspected incidents, or on the likelihood of incidents occurring (thus, when physical abuse was coded, this refers either to actual injuries or to social worker's views that physical abuse was likely given the history and circumstances). As Table 2 shows, neglect was the single most commonly identified concern-a finding consistent with that of the studies summarized by Brophy (2006, p. 6).

\begin{tabular}{|c|c|c|}
\hline Type of concern & $\begin{array}{c}\text { Number of children } \\
\text { (out of } 59 \text { ) }\end{array}$ & $\begin{array}{l}\text { Percentage of children to } \\
\text { nearest whole number }\end{array}$ \\
\hline Neglect & 50 & 85 \\
\hline Physical abuse & 35 & 59 \\
\hline Emotional abuse & 30 & 51 \\
\hline Domestic violence & 28 & 47 \\
\hline Child sexual abuse & 14 & 23 \\
\hline
\end{tabular}

\section{Pre-court delay: definition and problems with definition}

We now move on to look at the main subject of this article. By 'pre-court delay', we mean any kind of delay in the process of decision making prior to a child's case coming to court, which results in the child's future being resolved more slowly than would be in the child's best interests. A considerable period between serious concerns being raised and going to court is, of course, not necessarily delay at all and may be entirely appropriate, since it is a very serious step to enter the court arena and not one to be taken unless other options have been exhausted. However, the following are some instances of cases that seemed to us, on the evidence available to us, and/or to the current social workers, to have been slow in coming to court:

Case $\mathrm{Y}$ concerned a child of eight-and-a-half. This was the second set of proceedings on this child. He was returned home after the first set of proceedings on the recommendation of a psychologist, though against the view of the guardian. 'The nettle should have been grasped then' was the feeling of the social worker who initiated the second set, four years on. The current proceedings were not initiated as a result of a single event but as the result of this social worker compiling a chronology and seeing how bad things looked when events were seen together rather than in isolation.

Case C involved two children, one aged nearly four, one aged five-and-a-half. There was a list of concerns stretching back four years prior to the initiation of these care proceedings, including concerns about neglect, exposure to domestic violence and parental mental illness. The social worker in this case felt that it had taken longer to enter the court arena than would have been in the children's best interests as a result of: (i) use of 'accommodation' (removal of children from home on a voluntary basis) for four months, during which little progress was made with the case, (ii) previous handling of the case involving several cycles of referralinvestigation-closure with little in the way of substantive intervention, and (iii) psychiatric workers being over-optimistic about the mother's ability to care for the children.

Case S involved children aged nearly two and five. This was a case of serious neglect: the five-year-old had been found wandering the streets on her own, faeces were observed on toys, children were seen attempting to feed themselves and eating food sitting in urine, and 
the younger child had speech delay of one year. After an initial assessment, the case was referred to a non-statutory agency and closed. Although the mother did not engage with this agency and the health visitor made several attempts to get the local authority to reconsider its decision not to take on the case, it was not picked up again for another eight months.

Case $\mathrm{X}$ involved a boy aged ten and a girl aged one. There were longstanding problems going back eight years, with an older child having, on several occasions, 'voted with his feet' to get out, taking himself to grandparents and refusing to have contact with parents. There were significant issues of parental drinking and domestic violence. There was a history of repeated referrals that were dealt with by 'No further action' or minimal responses.

Case I involved a large sibling group aged from newborn to eight. There was concern about large number of sexual offenders in and around the family, and symptoms of physical or sexual abuse including a scald mark, cigarette burns, an anal injury and bite marks. The children had been on the child protection register more than once. The social worker was critical of past failure to get hold of the seriousness of this case, which had a nine-year history of social work involvement. In the social worker's own phrase, it was a case of 'head in the sand: it might go away'.

We accept that a more detailed analysis of some of these cases might well lead to a conclusion that delay, as opposed to the inevitable passing of time while work was done and information gathered, did not occur. However, pre-court delay can in some cases be quite clear-cut. If there are serious concerns about a child and a significant period of time elapses without intensive active work towards a resolution, then pre-court delay is definitely taking place. This is particularly obvious in situations in which a decision has been actually reached to initiate court proceedings but in which several months elapsed before this decision was implemented. In Case Q (discussed below), there was a five-month time lapse. We were also notified of another case in which it had been agreed to start proceedings, but proceedings had in fact not started four months after the decision: the case did not come within the remit of our study as a result.

Pre-court delay is also obvious in cases in which serious concerns exist but which have not been allocated to a social worker who could drive it forward (as occurred in Case Q.) Precourt delay is clearly evident, too, in cases in which a child has been removed from a family home on a 'voluntary' basis (or, at any rate, on a basis not ordered by a court) but then spends a protracted period in a temporary care arrangement such as a foster-home, without any long-term plans being made. (Children waiting for long periods in supposedly temporary foster-care are a long-standing problem well documented since the work of Rowe and Lambert, 1973.) Thirty-seven per cent of children in our study were already in foster-care at the outset of proceedings, and the average duration of time spent in foster-care prior to the start of proceedings was fourteen weeks.

In Case $\mathrm{HH}$, a child was 'accommodated' (taken into public care on a voluntary basis) at one year old and formal proceedings did not begin for another year. The eventual care plan at the end of court proceedings was to involve a placement move away from the foster-family in which (as a result of the long period prior to court) the child had been placed for more than half his life.

In Case Q, a boy of seven was accommodated for eleven months prior to proceedings. A decision by the appropriate panel to initiate proceedings was made five months before they actually were initiated, because the team apparently did not have the capacity to take the decision forward. There was a long history of maternal rejection of this child and life-long concerns about neglect. His mother had, on a number of occasions, come into social work offices, asking for him to be accommodated. She had also left him with many other carers.

In Case $\mathrm{M}$, a child aged three was accommodated seven months before the initiation of proceedings. Issues here were neglect, emotional abuse and exposure to domestic violence. The child had been placed on the child protection register more than two years before the initiation of proceedings. 
Case $R$ involved two children aged one-and-a-half and eight months. A suspected nonaccidental injury occurred two weeks after the birth of the younger child. The children were accommodated for eight months prior to the initial hearing.

\section{Causes of pre-court delay: resource issues}

There are a number of reasons why pre-court delay might occur, including a number of psychological factors such as reluctance to take a painful step, perhaps due to overinvolvement with or sympathy for parents, anxiety management by responding to individual events but failing to grasp the emerging 'big picture', and/or being overly reassured by an initial step that reduces the immediate risk to a child, with the result that the case ceases to be the focus of attention. Psychological defences against anxiety-provoking realities are likely to be in play in any situation involving material as painful as that entailed in decisions about care proceedings (see Taylor et al., 2008), and always have the potential to result in dangerous decision making.

However, it is surely the case that psychological defences are likely to become far more powerful and more potentially dangerous in situations in which there are inadequate resources. If there is simply no staff time available to work with a family, then it is less stressful to persuade oneself that this work is not necessary than to live with the tension of knowing that it is necessary but not being able to do it. (It is precisely in situations like this-of intolerable anxiety coupled with an inability to do anything to change the external circumstances-that psychological defences such as denial come into play.)

We wish, therefore, to resist the common tendency to automatically ascribe problems of these kinds simply to 'poor practice' or 'mistakes' because, although we certainly encountered some judgements that we considered questionable, and heard social workers describe other judgements that they considered questionable, the evidence before us suggested that one of the main problems causing pre-court delay was inadequate resources to perform the task. The authors feel that the tendency to ignore the role of resources in discussions of this kind and to focus solely on 'bad practice' constitutes a form of evasion (Beckett, 2007). It can often be politically expedient, as well as psychologically comforting, to focus on the shortcomings of particular actors, rather than on the system as a whole. Having made these points, though, we do not wish to deny that there may well have been many instances of poor professional practice in these cases that other participants-children, parents and colleagues-would have identified, and that could not be laid at the door of resource difficulties. We had such concerns ourselves in some cases, and there are likely to have been other instances that our methodology did not allow us to pick up. But the fact remains that the standard of practice that it is possible to deliver, and the speed at which it is possible to deliver it, is nevertheless necessarily limited by the resources available to meet demand.

We offer our evidence for thinking that a significant part of the problem of pre-court delay can be attributed to resource difficulties in the following discussion, while making clear at the outset that our data do not allow us to comment on the reasons for the resource difficulties (e.g. on whether these resource difficulties reflected problems within the agency, problems in the wider local authority or problems to do with the allocation of resources by central government).

First, we encountered, as discussed, a case that had gone unallocated during the period after a child had been removed from the care of a parent and two cases in which, following an agency decision to pursue care proceedings, no action was taken over a period of months, apparently due to lack of staff time. Second, we encountered situations in which there appeared to have been a pattern of short-term response to significant concerns, followed by closure, followed by new concerns (see cases $C, I, X$ and $Y$, described above). This typically occurs in teams that are having difficulty in meeting the demands made upon them. Third, and importantly, we encountered social workers who described very large workloads and who were, by their own admission, leaving work undone and who clearly demonstrated signs of stress. 
The social worker for Case I, for instance, told us that she was given three new cases involving care proceedings at a point at which she was still many months behind in her recording of other cases also subject to care proceedings. In another team, a social worker (responsible for cases $\mathrm{Q}$ and $\mathrm{HH}$ ) described a workload that, at one point, included ten families with children on the child protection register, two looked after children placed out of area, and two cases subject to care proceedings.

In Case FF, a part-time social worker had three other sets of care proceedings running concurrently. She was also incidentally caring for a seriously ill relative. We contacted this social worker about another set of proceedings that we had erroneously been told had started within the time parameters of our study. We were told by the social worker that it had indeed been agreed by the relevant panel that proceedings should be initiated, but the social worker had come out of the panel meeting in tears. This was the second set of proceedings that she had been asked to initiate on the same day, and she did not feel able to take on both.

The social worker latterly responsible for Case EE had been given this case even though she was an intake worker (i.e. her brief was to provide an initial response to new referrals). She said that the intake section was itself below the staff strength it was supposed to have to meet its designated brief. 'You are being pressured all the time to keep in with timescales [for intake work] and yet you are given a long term case.' This was one of several long-term cases that she had been given and she said she was not the only one in this position in the section. Her understanding was that budgetary problems meant that vacancies were not being filled.

Acute problems caused by vacant posts were described to us particularly in the two teams dealing with Case I and with Cases $\mathrm{Q}$ and $\mathrm{HH}$. It is worth noting that placing additional pressures on social workers can exacerbate recruitment problems if they make the job increasingly unattractive, and can therefore be counter productive if the aim of those pressures was to reduce delay.

\section{Court takes time from pre-court}

The resources that any agency has for any given task are a function of (i) the total 'pot' available to the agency, (ii) the effectiveness of the agency's management of that 'pot' and (iii) the number of competing claims on it. This study does not allow us to comment on (i) or (ii) or on the full range of competing claims, but it was clear to us that one important competing claim was the demand made on social workers' time and energy by court work.

The demands made by court work were described to us as considerable, not only emotionally, but in terms of time. We asked social workers at each interview to estimate the amount of time they were currently putting into each case. The first social worker for Case $X$ reported in April 2005 that the case was taking ten to fifteen hours per week. In August, the case's second social worker reported that it was taking two days a week. In December, the same social worker said it was now taking up more hours than her whole paid working week, while, in March 2006, she told us she was spending two to three hours per week on the case. The estimated time spent on Case EE was five to seven hours per week at the first interview, a day a week at the second and third interviews and two days a week at the final interview. The social worker in Case $\mathrm{H}$ told us, four months into proceedings, that he did not think he would have time to progress with parallel planning, as he had seven other children from two other families currently in care proceedings.

The pressure caused by court work on several occasions took the form of anger at the demands of the UK government's then Protocol for Judicial Case Management (DCA, 2003), which attempted to address the problem of court delay by setting tight timeframes for various tasks. The social worker in Case I expressed considerable hostility towards the Protocol, saying bluntly that the timescales it sets 'are impossible'. When it was pointed out to her that she had in fact managed to complete the case within the timeframe, she said it was at too high a cost and that, since she had not done any recording on this case since some nine months previously, she had only kept within the timescales by cutting corners with her practice. 


\section{Discussion}

Changes came into effect in April 2008, aimed at reducing delay in the court process. They had two interconnected strands. First, there were new case management procedures, set out in the Public Law Outline (PLO) (Ministry of Justice, 2008), which replaced the previous Protocol. They place much greater emphasis on pre-proceedings preparation than has been the case up to now, with the aim of ensuring that applications are made only in appropriate circumstances and only after all other options have been properly explored. One significant change from the Protocol is that the forty-week target is now replaced by an individual timetable for every child - a recognition that some cases can be dealt with in less than forty weeks. (We welcome this, as we found several examples in the present study in which the forty-week target of the protocol had the perverse effect of acting as a disincentive to speedier conclusion.)

The second strand consists of revised statutory guidance to local authorities (DCSF, 2008) setting out what local authorities need to have done pre-court in order to avoid unnecessary delay during the court process. This includes completion of core assessments, currently not done in one-third of cases that enter the court arena (Brophy, 2006), exploration of all kinship options and preparing and sharing care plans with parents.

We have not seen any published results of the pilot studies associated with these recent developments that were carried out in 2007, so have no direct evidence about the effect these changes have had on delay in the initiative areas. Given the short timeframe allocated to the feedback process, it may be that it is too early to tell. However, we are concerned that the changes may do no more than shift delay from one part of the process (during court) to another (pre-court) - a concern also voiced by an expert witness in a recent article (Taylor, 2007, pp. 16-19). In the discussion earlier, we described two cases in which a decision that it was appropriate to implement care proceedings had been taken by a local authority but was not progressed for several months due to lack of staff time. Would such delays not get even longer and/or more frequent if the amount of work required to bring a case to court was even greater? We have also described cases in which children were placed in accommodation (voluntary care) sometimes for as long as a year, and, in one case, with no allocated social worker for a period. Would there be any benefit to children if time in care under the supervision of a court was reduced, only for pre-court time in care, with no court supervision, to increase?

The government's optimistic view seems to be that the burden of work for local authorities will remain the same or 'may reduce' as compared to that under the previous Protocol, because it involves "front loading" interventions rather than . . . adding additional stages to the process' (Ministry of Justice, undated, p. 6). However, at this stage, it is impossible for anyone to say whether this is correct. It may be that an increased burden of work is the actual result. Even supposing that the total amount of work remains the same, any reductions in delay during proceedings might be offset by delays pre-court, if core assessments and kinship assessments were to back up and local authorities resorted to the various strategies discussed earlier (such as the use of 'accommodation') as a means of managing the flow of work.

In contrast to the government, the Magistrates Association Family Proceedings Committee does foresee problems for local authorities. Their proposed solution, however, lies in courts having appropriate sanctions to deal with recalcitrant authorities:

Currently, considerable delay is caused by Local Authorities-high turnover of staff, too many assessments not completed on time, reliance on long and repetitive reports and chronologies, drift, control of case left to guardian-which active case management has been unable to control. The identification and focus on issues requires a complete change in culture by the social workers [our italics]. The court will control this by active case management but we would welcome guidance as to effective action on non compliance as without sanctions, this may be difficult to achieve (Magistrates' Association, 2007, p. 2). 
It is instructive to contrast this approach with the concerns the Magistrates Association had about the impact of the changes on themselves:

The FPCs are dependent on resources (adequate Legal Advisers, admin staff, available user friendly courtrooms) and failure to provide these will lead to inevitable delay and failure to deliver (Magistrates' Association, 2007, p. 4).

The rather obvious double standard neatly illustrates the point we made earlier, when discussing the methodology of this study, that every group of participants is particularly well placed to describe the constraints it works under, less well placed to understand or sympathize with the constraints under which others labour. The response of the Association of Lawyers for Children seems, however, to take a more realistic view of the position of social work agencies, which is consistent with national reports (see, e.g. Hill, 2007) of recruitment and retention problems in this area of social work:

Social Services Departments continued to be seriously understaffed, suffering both recruitment and staff retention problems. This critically limits their ability to speed up the pre-application stages of the process (Association of Lawyers for Children, 2007, p. 27).

There may be some situations in which sanctions might be appropriate, but, if the main problem is lack of time, sanctions might possibly squeeze the problem to other less closely scrutinized parts of the system, but will not resolve the fundamental issue. The accounts we gave above of the workloads of social workers suggested to us that their agency was indeed having real difficulties in meeting the demands placed upon it by the existing protocol and that work was backing up as a result.

Another issue that needs addressing, if the switch to pre-court preparation is to be successful, is the status of social work core assessments. We were told by several social workers in our study (and it is a topic that we hope to return to in a later article) that, even though they had provided core assessments at the start of proceedings, these were disregarded by the court. In several cases, we were told that parents' solicitors persuaded the court to agree to assessments being re-done by independent social workers, resulting in increased delay for children before their future can be settled. If social work assessments were to continue to be discounted in this way, it could be that additional work done pre-court would not only cause pre-court delay but it might not even reduce court delay.

We do not know if the lack of credence given to social work reports can all be attributed to the quality of those reports (as opposed, say, to the perceived low status of social workers as against other professionals) but high-quality assessments are crucial to the success of the new initiative and evidence from elsewhere suggests that currently these are very variable, some being very sophisticated and others very poor 'often very process oriented with little or no analysis' (Brophy, 2006, p. 42, citing views of child psychiatrists). Our methodology, as explained at the outset, does not provide evidence that would support or refute this.

\section{Conclusion}

We welcome efforts to reduce court delay but we are concerned that new attempts to tackle this problem may simply push delay back to the 'pre-court' stage, in which there are fewer checks and safeguards in place. We have presented evidence that worrying delay already occurs at that stage, partly due to the existing demands on social work time. In the introduction, we made an analogy with the practice of 'stacking' patients in ambulances so as to allow hospital trusts to meet their targets on waiting times. Since patients are worse off waiting in ambulances than they would be inside the hospital, and since these ambulances are also prevented from responding to new emergencies, this practice is likely to result in an overall deterioration of the service. We fear that children may similarly be worse off during an extended pre-court period, if this ends up meaning that they are left for longer periods in harmful home situations or accommodated for long periods in foster-homes with no clear time limits. 
We have also raised doubts here as to whether additional assessment work undertaken by local authority social workers will even necessarily be effective in reducing court delay, since evidence suggests that social work assessments have limited currency in the court arena, though we have acknowledged that poor assessment and report writing skills may be part of the problem, and one that might be addressed through training.

We suggest that the problem of children 'in limbo' requires equal and simultaneous attention to be given to the pre-court and post-court periods as is given to the court period itself. We also suggest that simply imposing new demands or sanctions aimed solely at the in-court period is unlikely, in most cases, to result in an overall reduction in damaging delays in decision making for children. Our data suggest to us that limited time is a major factor in precourt delay, and that if additional tasks are taken on in the pre-court period, as is now happening under the Public Law Outline, then pre-court delay may well become a still more serious problem, particularly bearing in mind the national shortage of children's social workers in the UK mentioned earlier. We do not deny that, in some cases, or even many cases, there may be poor practice that is not connected to resource shortfalls. But it seems to us likely that progress with reducing damaging delay across the board-pre-court, in-court and postcourt-may not be easy unless steps are taken that free up time. Politically difficult as they may all be, these might have to include at least one of the following: (i) additional resources for social work teams, (ii) steps that simplify the process as a whole and therefore reduce the demands made on the time of the professionals required to implement it, or (iii) raising the threshold of intervention so as to reduce the number of cases that come into the ambit of the court system.

\section{References}

Association of Lawyers for Children (2007) Response of the Association of Lawyers for Children to the Draft Public Law Outline, available online at http://www.alc.org.uk/docs/ALC Public Law Outline Sept 07.doc (accessed February 2008).

Beckett, C. (2001) 'Children who wait for courts', British Journal of Social Work, 31(2), pp. $317-24$

Beckett, C. (2007) 'The reality principle: Realism as an ethical obligation', Ethics and Social Welfare, 1(3), pp. 269-81.

Beckett, C. and McKeigue, B. (2003) 'Children in limbo: Cases where care proceedings have taken two years or more', Adoption and Fostering, 27(3), pp. 31-40.

Beckett, C., McKeigue, B. and Taylor, H. (2007) 'Coming to conclusions: Social work perceptions of the decision-making process in care proceedings', Child and Family Social Work, 12(1), pp. 54-63.

Bishop, S., Murphy, M., Jellinek, M., Quinn, Sister D.. and Poitrast, F. (1992) 'Protecting seriously mistreated children: Time delays in a court sample', Journal of Child Abuse and Neglect, 16(4), pp. 465-74.

Booth, Dame M. (1995) 'Delay in public law Children Act cases', Representing Children, 8(4), pp. $41-8$.

Brophy, J. (2006) Research Review: Child Care Proceedings under the Children Act 1989, London, DCA.

Brophy, J., Jhutti-Johal, J. and Owen, C. (2003) Significant Harm in a Multi-Cultural Setting, Research Series 1/03, London, DCA.

Campbell, D. (2008) 'Scandal of patients left for hours outside A\&E', The Observer, 17 February 2008, p. 1, available online at: http://www.guardian.co.uk/society/2008/feb/17/health.nhs1?gusrc=rss\&feed=networkf ront (accessed February 2008).

DCA (Department for Constitutional Affairs) (2003) Protocol for Judicial Case Management in Public LawChildren Act Cases, London,Department forConstitutional Affairs.

DCSF (Department for Children, Schools and Families) (2008) The Children Act 1989 Guidance and Regulations, Volume 1: Court Orders, London, Department for Children Schools and Families.

Goldstein, J., Freud, A. and Solnit, A. (1980) Beyond the Best Interests of the Child, New Edition, London, Burnett Books. 
Hill, A. (2007) 'Social worker crisis puts children at risk', The Observer, 7 October 2007, available online at http://www.guardian.co.uk/society/2007/oct/07/childrensservices.socialcare (accessed August 2008).

Hunt, J., Macleod, A. and Thomas, C. (1999) The Last Resort: Child Protection, the Courts and the 1989 Children Act, London, The Stationery Office.

Magistrates' Association (2007) Response to the Draft Public Law Outline, available online at http://www.magistrates-association.org.uk/documents/family proceedings/0768public-law-outline-response.doc (accessed February 2008).

McKeigue, B. and Beckett, C. (2004) 'Care Proceedings under the 1989 Children Act: Rhetoric and reality', British Journal of Social Work, 34(6), pp. 831-49.

McSherry, D., Iwaniec, D. and Larkin, E. (2004) Counting the Costs: The Children (Northern Ireland) Order (1995), Social Work and the Courts, Belfast, Queen's University.

Ministry of Justice (2008) Public Law Outline: Guide to Case Management in Public Law Proceedings, London, Ministry of Justice. Tackling Court Delay and Resultant PreCourt Delay Page 15 of 16

Ministry of Justice (undated) Statutory Guidance for Local Authorities and the Public Law Outline, Frequently asked Questions, available online at http://www.justice.gov.uk/docs/faqs-care-proceedings-reforms.pdf (accessed February 2008).

Rowe, J. and Lambert, L. (1973) Children Who Wait: A Study of Children Needing Substitute Families, London, Association of British Adoption and Fostering Agencies.

Seale, C. (2004) 'Coding and analysing data', in C. Seale, (ed.), Researching Society and Culture, 2nd edn, London, Sage, pp. 305-23.

Steinhauer, P. (1991) The Least Detrimental Alternative: A Systematic Guide to Case Planning and Decision-Making for Children in Care, Toronto, University of Toronto Press.

Taylor, A. (2007) Prepare for the Dock, Community Care, 1 November 2007, pp. 16-19.

Taylor, H., Beckett, C. and McKeigue, B. (2008) 'Judgements of Solomon: Anxiety and defences for social workers involved in care proceedings', Child and Family Social Work, 13(1), pp. 23-31.

Thomas, C., Murch, M. and Hunt, J. (1993) The Duration of Care Proceedings: Replication Study, London, HMSO. Page 16 of 16 Bridget McKeigue and Chris Beckett 